\title{
Arbeitsüberforderung und -unzufriedenheit von Lehrpersonen in der Schweiz National repräsentative Ergebnisse demografischer, klassen- und schulbezoge- ner Faktoren ${ }^{1}$
}

\section{Béat Windlin, Emmanuel Kuntsche, Marina Delgrande Jordan}

Anhand einer national-repräsentativen Stichprobe der Schweiz werden Zusammenhänge zwischen subjektiv wahrgenommener Arbeitsüberforderung bzw. -unzufriedenheit und demografischen, klassen- sowie schulbezogenen Faktoren untersucht, und Angaben von 534 Lehrpersonen der 5. bis 9. Schulstufe und deren Klassen mit multiplen linearen Regressionsmodellen analysiert. Es zeigt sich, dass Variablen, die sich auf problematisches Verhalten der unterrichteten Schulkinder beziehen, weder mit subjektiver Arbeitsüberforderung noch -unzufriedenheit der Lehrperson in Verbindung stehen. Dagegen scheinen neben demografischen Variablen die Unterstützung der Schule und der Eltern sowie weitere strukturelle Faktoren der Schule und der Klasse zur Erklärung von subjektiver Arbeitsüberforderung bzw. -unzufriedenheit von Lehrpersonen bedeutsam zu sein.

\section{Einleitung}

Zahlreiche epidemiologische Studien weisen darauf hin, dass Lehrerinnen und Lehrer in ihrem Beruf in besonderem Mass psychischen Belastungen ausgesetzt sind. Dadurch steigt die Gefährdung ihrer psychischen Gesundheit. Es besteht international Konsens, dass Lehrkräfte in diesem Zusammenhang als Risikogruppe betrachtet werden müssen (zusammenfassend: Freitag, 1998; Rudow, 1994; Schaarschmidt, 2005). In einer Schweizer Studie konnte ein relativ enger Zusammenhang zwischen beruflichen Belastungen und dem Auftreten sowohl psychoaffektiver als auch somatischer Beschwerden bei Lehrpersonen festgestellt werden (Delgrande Jordan, Kuntsche \& Sidler, 2005).

Im Rahmenmodell der Beanspruchung von Lehrkräften nach Rudow (1994) werden objektive Belastungsfaktoren durch die Arbeitsaufgaben und -bedingungen bestimmt. Diese sind als von der Lehrperson unabhängig gegebene Charakteristiken der Lehrtätigkeit in einer bestimmten Klasse einer bestimmten Schule zu verstehen. Objektive Belastungsfaktoren sind wertindifferent - erst wenn sie individuell von der Lehrperson kognitiv reflektiert werden, können sie als sub- 
jektive Belastungen relevant werden und sich in (positiven oder negativen) Befindlichkeiten äussern.

Viele Lehrerinnen und Lehrer berichten in ihrer Tätigkeit in dauerhafter Weise verschiedenartigen Belastungen ausgesetzt zu sein. Dazu zählen sowohl quantitative als auch qualitative Arbeitsüberforderung, etwa durch zu grosse Klassen, Disziplinprobleme seitens der Schulkinder, sowie fehlende Zeit für Vorbereitung und Durchführung des Unterrichts (zusammenfassend: Bachmann, 1999; Jarvis, 2002).

In der Schweiz wurde in verschiedenen, meist auf einzelne Kantone beschränkten Studien die psychische Belastung von Lehrpersonen thematisiert. In einer im Kanton Basel-Stadt durchgeführten Studie gaben 29\% der Lehrpersonen an, sich durch Arbeitsüberforderung ,stark' bis ,sehr stark' belastet zu fühlen (Ulich, Inversini \& Wülser, 2002). In mehreren Studien wurden die Lehrpersonen dahingehend befragt, wie sehr sie einzelne, spezifische Aspekte ihrer Arbeit als belastend empfinden. Es ist schwierig aus diesen Resultaten ein einheitliches Bild zu generieren. In einer in den Kantonen Bern und Zürich durchgeführten Studie erzielen Items, welche sich auf Auswirkungen von Schulreformen und auf administrative Arbeiten beziehen, die höchsten Werte. Erst an vierter Stelle folgt mit „Verhalten und Motivation mancher Schüler" ein Aspekt, der sich auf das Problemverhalten von Schulkindern bezieht (Albisser, Kirchhoff, Meier \& Grob, 2006). Demgegenüber wurden sowohl in einer Aargauer Studie als auch in den beiden bisherigen durchgeführten Erhebungswellen einer Thurgauer Studie von den Lehrpersonen unter einer Vielzahl verschiedenster Aspekte das „Verhalten schwieriger Schüler“ am häufigsten als starke Belastung empfunden (Nido, Akkermann, Ulich, Trachsler \& Brüggen, 2008; Trachsler et al., 2006; Trachsler, Ulich, Inversini \& Wülser, 2003).

In diesen Studien wurde auf die Analyse des Zusammenhangs zwischen einzelnen Belastungsaspekten und der allgemeinen empfundenen Berufsbelastung verzichtet. Bieri (2002) kam aber in einer älteren, zwischen 1994 und 1998 im Kanton Aargau erhobenen Untersuchung diesbezüglich zu einem interessanten Ergebnis: Er zeigte, dass Lehrpersonen des Problemverhalten von Schulkindern zwar als besonders belastend empfinden, entsprechende Items (Einschätzung der Belastung z.B. durch „unsoziales Verhalten der Schüler/innen“, „Gewalt und Aggressionen von Schüler/innen“) in linearen Regressionsmodellen aber dennoch keinen eigenständigen Beitrag zur Varianzaufklärung der allgemeinen Berufsbelastung leisten. Dieser Befund scheint darauf hinzudeuten, dass subjektiv als Belastung empfundene Aspekte der Arbeit nicht unbedingt als Einflussfaktoren auf die allgemeine Arbeitsbelastung gelten können.

Die Arbeits(un)zufriedenheit bildet zusammen mit der Arbeitsbelastung den Kernbereich der vorhandenen empirischen Untersuchungen zur beruflichen Gesamtsituation der Lehrpersonen. In seinem Grundmodell der Berufszufriedenheit geht Merz (1979) davon aus, dass Arbeitsunzufriedenheit durch eine Diskrepanz zwischen objektiven Arbeitsanforderungen und -bedingungen und individuellen Erwartungen und Ansprüchen entsteht. 
Die Arbeits(un)zufriedenheit wurde in vielen internationalen Studien als Indikator für die Einstellung gegenüber der Berufstätigkeit untersucht (zusammenfassend: Bachmann, 1999; Enzmann \& Kleiber, 1989; Ipfling, Peez \& Gamsjäger, 1995; Rudow, 1994; Schmitz, 1999; Spear, Gould \& Lee, 2000). Gemäss den Ergebnissen dieser Studien sind die meisten Lehrpersonen mit ihrem Beruf zufrieden. Sie schätzen vor allem den Arbeitsinhalt sowie die sozialen Kontakte und den pädagogischen Entscheidungsspielraum. Demgegenüber gelten u.a. Erziehungsprobleme und -misserfolge als Gründe für die Unzufriedenheit von Lehrpersonen mit ihrer Arbeit.

In der Schweiz durchgeführte Studien belegen ebenfalls, dass die meisten Lehrpersonen generell mit ihrem Arbeitsumfeld zufrieden sind. Sie sind mehrheitlich gerne Lehrerin bzw. Lehrer und finden ihren Beruf attraktiv (Bucher, 2001). In einer Waadtländer Studie gaben 79\% der befragten Lehrpersonen an, sich in ihrer beruflichen Tätigkeit verwirklichen zu können. 76\% würden wieder denselben Beruf ergreifen, wenn sie nochmals vor der Wahl stünden (Gonik, Kurth \& Boillat, 2000). Dieses Resultat entspricht einer im Auftrag des Dachverbands Schweizer Lehrerinnen und Lehrer 2006 in der Deutschschweiz durchgeführten Studie. Bei dieser wurden die Lehrerinnen und Lehrer ausserdem nach ihrer Zufriedenheit in Bezug auf verschiedene Aspekte ihrer beruflichen Tätigkeit befragt. Dabei wiesen die Lehrpersonen Items, die direkt mit dem Unterrichten und Erziehen zu tun haben, die höchsten Zufriedenheitswerte zu. Am unzufriedensten waren sie mit Aspekten, die administrative Arbeiten und die Umsetzung von Reformen betreffen (Landert, 2007).

Wie eingangs erwähnt können Arbeitsüberforderung und Arbeitsunzufriedenheit gravierende Konsequenzen für die Gesundheit von Lehrpersonen haben (Delgrande Jordan et al., 2005). Das psychische Wohlbefinden der Lehrpersonen ist aber nicht nur für deren eigene Gesundheit von grosser Wichtigkeit, sondern auch für das Wohlergehen der unterrichteten Schulkinder. Befragungen von Lehrpersonen und Schulkindern belegen, dass berufliches Engagement und eine tiefe Arbeitsbelastung auch in enger Verbindung mit der Unterrichtsqualität stehen (Klusmann, Kunter, Trautwein \& Baumert, 2006; Klusmann, Kunter, Trautwein, Lüdtke \& Baumert, 2008). Im Schnittpunkt zwischen Präventivmedizin und Pädagogik kommt der Erforschung relevanter Einflussfaktoren auf die subjektiv empfundene Arbeitsunzufriedenheit und -überforderung von Lehrpersonen deshalb eine besondere Bedeutung zu. Der vorangehende Literaturüberblick hat gezeigt, dass es an potentiellen Einflussfaktoren für die Arbeitsunzufriedenheit und -überforderung von Lehrpersonen nicht mangelt. In bisherigen Schweizer Studien wurden diese aber meist nur univariat beschrieben; $\mathrm{Zu}$ sammenhänge zwischen objektiven Belastungsfaktoren und der subjektiv wahrgenommenen Arbeitsunzufriedenheit und -überforderung wurden aber nur auf regional beschränkter Ebene erforscht (Bieri, 2002; Landert, 2007).

Diese explorativ angelegte Arbeit untersucht nun erstmalig für die Schweiz anhand einer national-repräsentativen Stichprobe potentielle Einflussfaktoren 
der subjektiven Arbeitsunzufriedenheit und -überforderung von Lehrpersonen. Als Indikatoren für die abhängigen Variablen der Arbeitsunzufriedenheit und -überforderung dienen die entsprechenden von Enzmann und Kleiber (1989) entwickelten Indizes. Diese sind als unterscheidbare Dimensionen der subjektiven Einschätzung der Lehrperson ihrer beruflichen Belastung in der konkreten Berufssituation zu verstehen.

Als unabhängige Variablen gehen neben demografischen Merkmalen der Lehrperson verschiedene objektive Belastungsfaktoren in die Untersuchung ein, nämlich klassenbezogen Variablen auf der Ebene der Schulklasse, sowie schulbezogene Variablen auf der Ebene der Schule als Institution.

Bei den klassenbezogenen Variablen wird zudem zwischen strukturellen Variablen (z.B. Klassengrösse) und verhaltensbezogenen Variablen differenziert. In Anlehnung an Klusmann et al. $(2006,2008)$ verfolgt die vorliegende Arbeit hier einen methodisch innovativen Ansatz, indem sie verhaltensbezogene Variablen der Schulklasse einschliesst, die auf der Befragung der Schulkinder basieren und somit von der subjektiven Einschätzung der Lehrperson unabhängig sind. Es soll somit insbesondere die gemäss der Literatur umstrittene Frage beantwortet werden können, ob die Arbeitsunzufriedenheit und -überforderung von Lehrkräften auch mit verhaltensbezogenen Merkmalen der unterrichteten Schulkinder in Zusammenhang stehen.

Für die Analysen werden Daten einer Lehrpersonen- und Schülerbefragung benutzt, welche im Rahmen der Studie „Health Behaviour in School-Aged Children" (HBSC) 2006 von der Schweizerischen Fachstelle für Alkohol- und andere Drogenprobleme (heute Sucht Info Schweiz) erhoben wurden.

\section{Methode}

\section{Studienbeschreibung}

Für die HBSC-Studie im Jahr 2006 wurden aus allen Landesteilen der Schweiz 690 Schulklassen zwischen dem 5. und 9. Schuljahr zufällig ausgewählt. Die Zufallsauswahl der Klassen und ihrer Lehrpersonen wurde unter Verwendung des Verzeichnisses des Bundesamts für Statistik vorgenommen, in dem alle Klassen der öffentlichen Schulen der Schweiz aufgelistet sind.

Sowohl die Schulkinder wie auch die Lehrperson wurden anhand eines standardisierten Fragebogens befragt. Der Lehrpersonenfragebogen umfasste Fragen zur Arbeitssituation, zum Schulumfeld, zur Einschätzung der Schulklasse und zum persönlichen Gesundheitsverhalten (siehe auch Delgrande Jordan et al., 2005; Kuntsche, Delgrande Jordan \& Sidler, 2005). Im Schulkinderfragebogen wurde ein breites Spektrum von Verhaltens- und Lebensweisen sowie das gesundheitliche Befinden der Jugendlichen erfasst (siehe auch Schmid, Kuntsche \& Delgrande, 2001; sowie www.hbsc.ch) 
Der Lehrperson und den Schülerinnen und Schülern stand eine Schulstunde zur Verfügung, um den Fragebogen auszufüllen. Die Teilnahme an der Befragung war für Schulkinder und Lehrkräfte freiwillig, und die resultierenden Daten wurden anonym und streng vertraulich behandelt. Die Daten wurden zwischen Januar und April 2006 erhoben.

\section{Subjektiv wahrgenommene Belastungen (abhängige Variablen)}

Die beiden Skalen Arbeitsüberforderung und Arbeitsunzufriedenheit von Enzmann und Kleiber (1989) erfassen zwei Dimensionen der subjektiv wahrgenommenen Belastung in der konkreten beruflichen Tätigkeit. Beide Skalen bestehen aus je sechs Items, die jeweils ein fünfstufiges Antwortformat haben $(1=$ „trifft gar nicht zu“, 2 = „trifft wenig zu“, 3 = „trifft mittelmäßig zu“, 4 = ,trifft überwiegend zu“ und $5=$, trifft völlig zu“) und sich im schulischen Kontext als praktikable und zuverlässige Erhebungsinstrumente erwiesen haben (Delgrande Jordan et al., 2005; Schwarzer \& Jerusalem, 1999). ${ }^{2}$

Die Indizes wurden gebildet indem die sechs Items jeweils aufsummiert und dann durch die Anzahl einbezogener Items (=6) dividiert wurden. Für den Index der Arbeitsunzufriedenheit wurden die fünf positiv formulierten Items vor der Indexbildung invertiert. Die beiden Indizes können Werte zwischen 1 (tiefe Arbeitsunzufriedenheit bzw. überforderung) und 5 (hohe Arbeitsunzufriedenheit bzw. -überforderung) annehmen.

Delgrande Jordan et al. (2005) zeigten, dass die Indizes der Arbeitsüberforderung und -unzufriedenheit nach Enzmann und Kleiber (1989) auch in der Schweiz ein valides Erfassungsinstrument darstellen. In einer Hauptkomponentenanalyse mit HBSC-Daten von 2002 konnten sie in Übereinstimmung mit anderen Forschungsergebnissen Arbeitsüberforderung und -unzufriedenheit als je separate Dimension identifizieren.

\section{Demografische Variablen}

Als individuelle Faktoren gehen das Geschlecht und das Alter der Lehrpersonen in diese Untersuchung ein. Das Geschlecht wurde mit 1 für Frauen und 0 für Männer codiert. Das Alter wurde im Lehrpersonenfragebogen in Kategorien von je fünf Jahren erfasst, wobei die unterste ,jünger als 26 “ und die oberste ,älter als 65" lautete. Die unterste Kategorie wurde mit 23 und die mittleren Alterskategorien je mit ihrem Mittelwert recodiert. Die oberste Alterskategorie wurde von keiner in der hier verwendeten Stichprobe enthaltenen Lehrperson angegeben.

Aufgrund der relativ kleinen Gruppengrösse wurden die italienisch- und die französischsprachige Schweiz zur lateinischen Schweiz zusammengefasst. Die Variable der Sprachregion wurde mit dem Wert 0 für die Deutschschweiz und dem Wert 1 für die lateinische Schweiz recodiert. 
Potentielle Belastungsfaktoren: Klassenbezogene Variablen Die Schulstufe der Klasse wurde aufgrund der Angaben der Lehrperson bestimmt. Doppel- oder Mehrfachklassen wurden dabei als Mittel der darin vertretenen Schulstufen codiert. Als Näherung für die Klassengrösse wurde die Anzahl der Schulkinder verwendet, die an der Befragung teilgenommen haben.

Die verhaltensbezogenen Variablen der Schulklassen beruhen auf den Angaben von insgesamt 9'867 Schulkindern der in die Analysen einbezogenen Klassen. Folgende Fragen wurden hier betrachtet:

- „Hast du jemals Tabak (eine Zigarette, Zigarre oder Pfeife) geraucht?“

- „Warst du schon einmal richtig betrunken?"

- „Wie oft bist du in den letzten paar Monaten in der Schule schikaniert oder geplagt worden?“

Die Antworten der Schülerinnen und Schüler wurden auf Klassenebene aggregiert. Dabei wurde zu jeder Frage auf der Basis der gegebenen Antworten der Anteil der Schulkinder berechnet, die angaben, bereits geraucht zu haben, schon einmal betrunken gewesen zu sein bzw. in den letzten Monaten mindestens einmal schikaniert worden zu sein. Im Durchschnitt lag der Anteil gegebener Antworten pro Klasse bei der 1. und 3. Frage bei über 99\% und bei der 2. Frage bei $96.1 \%$.

Diese aggregierten verhaltensbezogenen Variablen der Schulklasse geben zwar nicht direkt das Verhalten der Schulkinder im Klassenzimmer und während des Unterrichts wieder. Sie weisen aber auf das Vorhandensein und Ausmass problematischer Verhaltensweisen unter den Schülerinnen und Schülern der unterrichteten Klasse hin (Jessor \& Jessor, 1977; Kuntsche, 2004) und werden hier als objektiver Belastungsfaktor gewertet. Gegenüber entsprechenden Angaben der Lehrerinnen und Lehrer haben sie den Vorteil, dass bei ihnen eine rekursive Beeinflussung durch den Grad der subjektiv wahrgenommenen Arbeitsunzufriedenheit oder -überforderung der Lehrperson praktisch ausgeschlossen werden kann.

\section{Potentielle Belastungsfaktoren: Schulbezogene Variablen}

Die erste schulbezogene Variable bezieht sich auf die institutionelle Unterstützung, welche Lehrkräfte von Seiten der Schule zur Bewältigung einer über die reine Wissensvermittlung hinausgehenden Aufgabe erfahren. Als Indikator hierfür wird der Grad der Zustimmung der Lehrperson auf die Aussage „die Schule unterstützt die Anstrengungen der Lehrpersonen in der Gesundheitserziehung" verwendet. Die möglichen Antwortkategorien entsprechen denjenigen der oben vorgestellten Items, die zur Bildung der Indizes der Arbeitsunzufriedenheit und -überforderung herangezogen wurden, ebenfalls mit möglichen Werten von 1 bis 5 .

Die zweite schulbezogene Variable bezieht sich auf die institutionalisierte $\mathrm{Zu}$ sammenarbeit zwischen der Schule und den Eltern, bzw. auf die Unterstützung, welche die Lehrpersonen in ihrem Schulalltag daraus ziehen können. Hierzu wurden die Antworten der Lehrpersonen auf die Aussage „die Eltern unterstützen die Schule in den Bereichen Erziehung und Gesundheitsförderung"verwen- 
det. Dabei standen den Lehrerinnen und Lehrern folgende drei Antwortkategorien zur Verfügung: „trifft völlig zu“, „trifft mittelmässig zu“ und „trifft gar nicht zu“. Da nur sehr wenige Lehrpersonen (6.9\%) die dritte Antwortkategorie („trifft gar nicht zu“) wählten, wurde sie zusammen mit der mittleren („trifft mittelmässig zu“) mit dem Wert 0 als ,Ablehnung', und die erste („trifft völlig zu“) mit dem Wert 1 als ,Zustimmung' recodiert.

\section{Stichprobenbeschreibung und Ersetzung fehlender Werte}

Von den 690 zufällig ausgewählten Klassen haben 591 an der Untersuchung teilgenommen. Davon haben in 570 Fällen auch die Lehrpersonen geantwortet. Daraus ergibt sich für die Lehrpersonenbefragung eine Rücklaufquote von 82.6\% bezogen auf die ausgewählten Klassen und von $96.4 \%$ bezogen auf die teilnehmenden Klassen. Die Auswahl kann als repräsentativ für die Lehrpersonen der 5. bis 9. Klassen an öffentlichen Schulen in der gesamten Schweiz gelten.

Ein Erkenntnisinteresse dieser Untersuchung richtet sich auf die Frage, ob die Arbeitsüberforderung bzw. -unzufriedenheit einer Lehrperson mit klassenspezifischen Faktoren in Verbindung steht. Um zu gewährleisten, dass es zwischen der befragten Lehrperson und der entsprechenden Klasse zu einer regelmässigen Interaktion kommt - und somit die Möglichkeit zu einer potentiellen Beeinflussung der Arbeitsunzufriedenheit bzw. -überforderung gegeben ist - wurden 13 Lehrpersonen (2.2\%) aus der Untersuchung ausgeschlossen, welche die befragte Klasse normalerweise nicht unterrichten. Weiter wurde die Lehrperson einer Klasse ausgeschlossen, in der weniger als 10\% der Schulkinder die Fragen zu den hier verwendeten klassenspezifischen Faktoren beantwortet haben. Zwei Lehrpersonen ohne Angaben zu ihrem Geschlecht und Alter sowie 20 weitere Lehrerinnen und Lehrer (3.5\%) mit fehlenden Angaben bei einem oder mehreren der zwölf Items, aus denen die Indizes der abhängigen Variablen berechnet werden, mussten ebenfalls aus den Analysen ausgeschlossen werden.

Die resultierende Stichprobe enthält 534 Lehrpersonen. Darin sind die Männer in der Überzahl (Männer: 60.7\%; Frauen: 39.3\%). Die Verteilung auf die Sprachregionen (Deutsch: 72.7\%; Französisch: 21.7\%; Italienisch: 5.6\%) unterstreicht die Repräsentativität der Stichprobe auf nationaler Ebene.

Bei den beiden schulbezogenen Variablen wurden fehlende Angaben mit Hilfe des Markov Chain Monte Carlo (MCMC) Verfahrens ersetzt (Gilks, Richardson \& Spiegelhalter, 1996; Schafer, 1997). Der Vorteil dieses Verfahrens liegt darin, dass für das Ersetzen der fehlenden Antworten eines Individuums die Information seines übrigen Antwortschemas und das aller anderen Personen in der Stichprobe miteinbezogen wird. Das MCMC-Verfahren wurde mit dem Programm LISREL 8.51 (Jöreskog \& Sörbom, 2001) durchgeführt. Bezüglich der Unterstützung der Eltern und der Unterstützung der Schule wurden insgesamt fehlende Angaben von 45 Lehrpersonen (8.4\%) ersetzt. 


\section{Statistische Analysen}

Zur Bestimmung der Zusammenhänge zwischen demografischen, schul- und klassenbezogenen Variablen und Arbeitsüberforderung und -unzufriedenheit wurden lineare multiple Regressionsanalysen verwendet. In einem ersten Modell wurden jeweils für die Arbeitsunzufriedenheit und -überforderung getrennt $\mathrm{Zu}$ sammenhänge mit allen oben beschriebenen unabhängigen Variablen geschätzt.

Um zu überprüfen, ob unterschiedliche Zusammenhänge in den Alters- und Geschlechtsgruppen oder in den Sprachregionen vorliegen, wurden in einem zweiten Schritt entsprechende Interaktionen in das Regressionsmodell aufgenommen. Hierzu wurden Interaktionsterme als Produkt je der genannten demografischen mit den restlichen unabhängigen Variablen berechnet (Jaccard, Turrisi \& Wan, 1990). Daraus resultieren 24 Interaktionsterme.

Aufgrund ihrer hohen Anzahl wurde darauf verzichtet, alle Interaktionsterme gleichzeitig in die Regressionsmodelle aufzunehmen. Stattdessen wurde zur Selektion relevanter Interaktionsterme die Vorwärtsmethode verwendet. Durch diese Methode wurde schrittweise derjenige Interaktionsterm zusätzlich in das Modell aufgenommen, der am stärksten zu einer Steigerung der Varianzaufklärung $\left(\mathrm{R}^{2}\right)$ beitragen konnte. Dabei wurde vorausgesetzt, dass das Signifikanzniveau des FWertes seines partiellen Korrelationskoeffizienten den Schwellenwert $\mathrm{p}=0.05$ unterschreiten musste. Erfüllte kein weiterer Interaktionsterm dieses Kriterium, wurde das Verfahren gestoppt (Backhaus, Erichson, Plinke \& Weiber, 2000).

In dieser Arbeit wird als Gesamtvarianzaufklärung generell das nach Stichprobengrösse und Anzahl Variablen korrigierte $\mathrm{R}^{2}$ berichtet.

\section{Ergebnisse}

Tabelle 1 fasst die Verteilungen der nicht aggregierten unabhängigen Variablen zusammen. Für Variablen qualitativer Merkmale werden die prozentualen Verteilungen in der verwendeten Stichprobe und bei quantitativen Merkmalen der Mittelwert und die Standardabweichung angegeben. Da sich bei den aus den Angaben der Schulkinder berechneten, verhaltensbezogenen Variablen der Schulklassen die Anteile zwischen den Schulstufen erwartungsgemäss stark unterscheiden, werden diese in Tabelle 2 nach Klassenstufen unterteilt separat dargestellt. Es wird dabei klar ersichtlich, dass der Anteil der Schulkinder, die schon einmal geraucht haben oder betrunken gewesen sind, mit der Höhe der Schulstufe zunimmt $(\mathrm{r}=0.75$ bzw. 0.69, $\mathrm{p}<0.001$ für beide). Der Anteil der Schulkinder, die angaben, in den letzten paar Monaten vor der Befragung schikaniert worden zu sein, nimmt dagegen $a b(r=-0.31, p<0.001)$. Die hohen Standardabweichungen verweisen aber auf eine hohe Variation der Anteile auch innerhalb einer gegebenen Klassenstufe. 
Tab. 1: Übersicht unabhängige Variablen (ohne verhaltensbezogene Variablen der Schulklassen)

\begin{tabular}{|c|c|c|c|}
\hline & $\mathrm{n}$ & $\begin{array}{c}\text { relative } \\
\text { Häufigkeit }\end{array}$ & $\begin{array}{l}\text { Mittelwert } \\
\text { (Standard- } \\
\text { abweichung) }\end{array}$ \\
\hline \multicolumn{4}{|l|}{ Demografische Variablen: } \\
\hline \multicolumn{4}{|l|}{ Geschlecht } \\
\hline Männlich & 324 & $60.7 \%$ & \\
\hline Weiblich & 210 & $39.3 \%$ & \\
\hline Alter & 534 & & $42.6(11.4)$ \\
\hline \multicolumn{4}{|l|}{ Sprachregionen } \\
\hline Deutschschweiz & 388 & $72.7 \%$ & \\
\hline Lateinische Schweiz & 146 & $27.3 \%$ & \\
\hline \multicolumn{4}{|l|}{ Klassenbezogene Variablen: } \\
\hline \multicolumn{4}{|l|}{ Schulstufe ${ }^{a}$} \\
\hline 5. Klassen & 104 & $19.5 \%$ & \\
\hline 6. Klassen & 106 & $19.9 \%$ & \\
\hline 7. Klassen & 110 & $20.6 \%$ & \\
\hline 8. Klassen & 104 & $19.5 \%$ & \\
\hline 9. Klassen & 110 & $20.6 \%$ & \\
\hline Klassengrösse & 534 & & $18.5(3.8)$ \\
\hline \multicolumn{4}{|l|}{ Schulbezogene Variablen: } \\
\hline \multicolumn{4}{|l|}{ Unterstützung der Eltern } \\
\hline Zustimmung & 134 & $25.1 \%$ & \\
\hline Ablehnung & 400 & $74.9 \%$ & \\
\hline $\begin{array}{l}\text { Unterstützung der Schule } \\
\text { (Skala 1-5) }\end{array}$ & 534 & & $3.74(0.95)$ \\
\hline
\end{tabular}

a Bei Doppel- und Mehrfachklassen wurde der Mittelwert der darin enthaltenen Stufen gebildet und die ganze Klasse der entsprechenden Schulstufe zugeordnet. Aus Darstellungsgründen wurden dabei nicht geradezahlige Mittelwerte abgerundet und ebenfalls der entsprechenden Stufe zugeordnet. 
Tab. 2: Übersicht verhaltensbezogene Variablen der Schulklassen (durchschnittliche Anteile nach Schulstufen in Prozent, Standardabweichung in Klammern)

\begin{tabular}{|l|c|c|c|}
\hline & \multicolumn{3}{|c|}{ Anteil der Schülerinnen und Schüler, die... } \\
\hline Schulstufe $^{\mathrm{a}}$ & $\begin{array}{c}\text {...schon einmal } \\
\text { geraucht haben }\end{array}$ & $\begin{array}{c}\text {...schon einmal } \\
\text { betrunken waren }\end{array}$ & $\begin{array}{c}\text {...schikaniert } \\
\text { worden sind }\end{array}$ \\
\hline 5. Klassen (n=104) & $12.8 \%(11.1)$ & $5.9 \%(7.9)$ & $42.4 \%(15.1)$ \\
\hline 6. Klassen (n=106) & $19.9 \%(14.6)$ & $9.7 \%(9.1)$ & $42.5 \%(16.0)$ \\
\hline 7. Klassen ( $\mathrm{n}=110)$ & $32.9 \%(15.6)$ & $16.1 \%(11.5)$ & $41.4 \%(15.1)$ \\
\hline 8. Klassen ( $\mathrm{n}=104)$ & $47.1 \%(16.0)$ & $26.9 \%(14.6)$ & $32.7 \%(15.0)$ \\
\hline 9. Klassen (n=110) & $59.9 \%(18.6)$ & $40.6 \%(16.5)$ & $30.1 \%(13.5)$ \\
\hline
\end{tabular}

Anmerkungen: Die Standardabweichung bezieht sich auf Prozentpunkte.

a Bei Doppel- und Mehrfachklassen wurde der Mittelwert der darin enthaltenen Stufen gebildet und nicht geradezahlige Mittelwerte abgerundet.

Der Index der Arbeitsunzufriedenheit hat in der verwendeten Stichprobe einen Durchschnittswert von 2.06 und einen Median von 2.00 (unteres Quartil: 1.67, oberes Quartil: 2.50, Standardabweichung: 0.58). Der Durchschnitt des Index der Arbeitsüberforderung liegt bei 2.28, sein Median bei 2.17 (unteres Quartil: 1.83, oberes Quartil: 2.67, Standardabweichung: 0.72). Führt man sich die Antwortkategorien auf die den Indizes zugrunde liegenden Items vor Augen, bedeutet dies, dass die meisten Lehrpersonen auf die Mehrzahl der Fragen mit "trifft gar nicht zu“ $(=1)$, ,trifft wenig zu“ (=2) oder „trifft mittelmässig zu“ (=3) geantwortet haben. ${ }^{3}$ Lediglich $4.9 \%$ der Lehrpersonen weisen bei der Arbeitsunzufriedenheit einen höheren Indexwert als 3 auf. Bei der Arbeitsüberforderung sind es $13.5 \%$.

Tabelle 3 zeigt die durchgeführten linearen Regressionsanalysen mit dem Index der Arbeitsunzufriedenheit bzw. -überforderung als abhängige Variable. In den Spalten 1a) und 2a) sind die Standardmodelle mit allen oben eingeführten unabhängigen Variablen, aber ohne Interaktionsterme wiedergegeben. In den Spalten $1 \mathrm{~b}$ ) und 2b) finden sich die Modelle, bei denen zum Standardmodell mittels der Vorwärtsmethode ausgewählte Interaktionsterme hinzugefügt wurden. Bei der Arbeitsunzufriedenheit wurden die Interaktionsterme zwischen der Sprachregion und der Unterstützung der Schule und zwischen dem Geschlecht und der Klassengrösse in das Modell aufgenommen. Bei der Arbeitsüberforderung erreichte nur der Interaktionsterm zwischen Alter und der Unterstützung der Eltern das Aufnahmekriterium.

Wenden wir uns zunächst den Standardmodellen in den Spalten 1a) und 2a) zu: Es fällt auf, dass die klassenbezogenen Indikatoren für problematisches Verhalten der unterrichteten Schulkinder keinen signifikanten Zusammenhang mit der subjektiv wahrgenommenen Arbeitsunzufriedenheit oder -überforderung der Lehrperson aufweisen. Dies gilt sowohl für die Anteile der Schulkinder, die 
schon geraucht haben oder betrunken waren, als auch für jenen der Schülerinnen und Schüler, die schikaniert worden sind.

Tab. 3: Zusammenhänge der subjektiv wahrgenommenen Arbeitsunzufriedenheit und überforderung von Lehrpersonen mit demografischen, klassenbezogenen und schulbezogenen Faktoren (unstandardisierte Regressionskoeffizienten mit Standardfehlern in Klammern) und Varianzaufklärung; $n=534$

\begin{tabular}{|c|c|c|c|c|}
\hline & 1a) & 1b) & 2a) & 2b) \\
\hline & \multicolumn{2}{|c|}{ Arbeitsunzufriedenheit } & \multicolumn{2}{|c|}{ Arbeitsüberforderung } \\
\hline \multicolumn{5}{|l|}{ Demografische Variablen: } \\
\hline Geschlecht $^{\mathrm{a}}$ & $-.060(.054)$ & $.577^{*}(.248)$ & $.066(.066)$ & $.060(.066)$ \\
\hline Alter & $.005^{*}(.002)$ & $.004+(.002)$ & $.005^{*}(.003)$ & $.009^{* *}(.003)$ \\
\hline Sprachregion ${ }^{b}$ & $.043(.059)$ & $.636^{* *}(.223)$ & $.350^{* * *}(.071)$ & $.357^{* * *}(.071)$ \\
\hline \multicolumn{5}{|l|}{ Klassenbezogene Variablen: } \\
\hline Schulstufe ${ }^{\mathrm{c}}$ & $-.059^{*}(.029)$ & $-.064^{*}(.029)$ & $-.102^{* *}(.035)$ & $-.105^{* *}(.035)$ \\
\hline Klassengrösse & $.001(.007)$ & $.015+(.008)$ & $.016+(.008)$ & $.016^{*}(.008)$ \\
\hline \multicolumn{5}{|l|}{ Anteil der Schulkinder, die... } \\
\hline $\begin{array}{l}\text {...schon einmal geraucht } \\
\text { haben }\end{array}$ & $.172(.198)$ & $.186(.197)$ & $.084(.240)$ & $.090(.240)$ \\
\hline $\begin{array}{l}\text {...schon einmal betrunken } \\
\text { waren }\end{array}$ & $-.047(.237)$ & $-.026(.237)$ & $.072(.287)$ & $.077(.286)$ \\
\hline ...schikaniert worden sind & $.261(.168)$ & $.245(.166)$ & $-.272(.204)$ & $-.275(.203)$ \\
\hline \multicolumn{5}{|l|}{ Schulbezogene Variablen: } \\
\hline Unterstützung der Eltern ${ }^{\mathrm{d}}$ & $.193^{* * *}(.058)$ & $.192^{* * *}(.058)$ & $.101(.071)$ & $.422(.263)$ \\
\hline Unterstützung der Schule & $.071^{* *}(.027)$ & $.025(.031)$ & $.016(.032)$ & $.017(.032)$ \\
\hline \multicolumn{5}{|l|}{ Interaktionsterme: } \\
\hline $\begin{array}{l}\text { Geschlecht }{ }^{\mathrm{a}} \mathrm{x} \\
\text { Klassengrösse }\end{array}$ & & $-.035^{* *}(.013)$ & & \\
\hline $\begin{array}{l}\text { Alter x Unterstützung der } \\
\text { Eltern }\end{array}$ & & & & $-.012^{*}(.006)$ \\
\hline $\begin{array}{l}\text { Sprachregion }{ }^{\mathrm{b}} \mathrm{x} \\
\text { Unterstützung der Schule }\end{array}$ & & $-.156^{* *}(.057)$ & & \\
\hline Korrigiertes $\mathrm{R}^{2}$ & $5.1 \%$ & $7.2 \%$ & $8.2 \%$ & $8.8 \%$ \\
\hline
\end{tabular}

Anmerkungen:

a Weiblich als 1, männlich als 0 codiert;

b Lateinische Schweiz als 1; Deutschschweiz als 0 codiert;

c Doppel- und Mehrfachklassen als Mittelwert der darin enthaltenen Stufen codiert;

d "Trifft völlig zu“ als 1 , „triff mittelmässig zu“ und „trifft gar nicht zu“ als 0 codiert.

$+\mathrm{p}<0.10$

${ }^{*} \mathrm{p}<0.05$

${ }^{* *} \mathrm{p}<0.01$,

*** $\mathrm{p}<0.001$ 
Arbeitsunzufriedenheit und -überforderung der Lehrpersonen stehen aber mit anderen Faktoren in Zusammenhang. Bei der Arbeitsunzufriedenheit (Spalte 1a) steht die von der Lehrperson wahrgenommene Unterstützung im Bereich Erziehung und Gesundheitsförderung bzw. Gesundheitserziehung durch die Eltern und die Schule in einem negativen Zusammenhang mit der subjektiv wahrgenommenen Arbeitsunzufriedenheit der Lehrerinnen und Lehrer: Lehrpersonen, die sich von den Eltern oder der Schule unterstützt fühlen, berichten eher eine tiefe Arbeitsunzufriedenheit.

Auch in Bezug auf die Schulstufe zeigt sich ein signifikanter Zusammenhang. Unabhängig von den weiteren strukturellen und individuellen Faktoren sind die Lehrpersonen tieferer Stufen tendenziell unzufriedener mit ihrem Arbeitsumfeld als ihre Kolleginnen und Kollegen höherer Stufen.

Von den demografischen Variablen hat einzig das Alter einen Einfluss auf die Arbeitsunzufriedenheit. Dieser ist positiv; ältere Lehrpersonen sind mit ihrem Arbeitsumfeld also eher unzufriedener als jüngere.

Bei der Arbeitsüberforderung (Spalte 2a) stellt sich von den im Standardmodell enthaltenen Variablen neben der Schulstufe vor allem die Sprachregion als gewichtiger Faktor heraus. Es zeigt sich, dass Lehrpersonen in der lateinischen Schweiz sich signifikant stärker von ihrer Arbeit überfordert fühlen als jene in der Deutschschweiz.

Während das Geschlecht einer Lehrperson in keinerlei Zusammenhang mit ihrer Arbeitsüberforderung zu stehen scheint, lässt sich beim Alter - wie bereits bei der Arbeitsunzufriedenheit - ein positiver Zusammenhang beobachten: Ältere Lehrkräfte fühlen sich eher überfordert als jüngere.

Wie die Arbeitsunzufriedenheit ist auch die Arbeitsüberforderung bei Lehrpersonen höherer Schulklassen tendenziell weniger verbreitet als bei Lehrkräften tieferer Schulstufen. Bei der Klassengrösse zeigt sich erwartungsgemäss ebenfalls ein positiver Zusammenhang: Lehrpersonen grösserer Klassen weisen eher eine höhere Arbeitsüberforderung auf als Lehrpersonen kleinerer Klassen. Dagegen scheinen die schulbezogenen Variablen mit der Arbeitsüberforderung in keinem Zusammenhang zu stehen.

Der Einbezug von Interaktionstermen in das Regressionsmodell für die Arbeitsunzufriedenheit (Spalte 1b) zeigt, dass die Unterstützung der Schule im Bereich Gesundheitserziehung in der lateinischen Schweiz auch mit der Arbeitsunzufriedenheit der Lehrpersonen in Zusammenhang steht. Ein komplexer Zusammenhang lässt sich auch bei der Grösse der Klasse beobachten: Es sind vor allem die Lehrer, die mit zunehmender Klassengrösse unzufriedener werden, während sich bei den Lehrerinnen eher ein umgekehrter Zusammenhang zeigt.

Die im Standardmodell 1a) enthaltenen Variablen erklären 5.1\% der Varianz der Arbeitsunzufriedenheit. Dieser Wert steigt unter Einbezug der zwei Interaktionsterme auf $7.2 \%$.

Bei der Arbeitsüberforderung zeigt sich im Modell mit Interaktionsthermen (Spalte 2b), dass bei älteren Lehrpersonen auch die wahrgenommene Unterstüt- 
zung von Seiten der Eltern von Bedeutung zu sein scheint: Ältere Lehrpersonen, die berichten, dass sie von den Eltern in den Bereichen Erziehung und Gesundheitsförderung unterstützt werden, geben eine signifikant tiefere Arbeitsüberforderung an als Lehrpersonen derselben Altersgruppe, die keine solche Unterstützung berichten. Mit Einbezug dieses Interaktionsterms beträgt die Varianzaufklärung des Index der Arbeitsüberforderung 8.8\%, gegenüber $8.2 \%$ im Standardmodell 2a).

\section{Diskussion}

Das Ziel dieser explorativen Arbeit war es, mögliche Zusammenhänge zwischen der subjektiv wahrgenommenen Arbeitsunzufriedenheit bzw. -überforderung von Lehrpersonen in der Schweiz und verschiedenen demografischen Merkmalen und objektiven Belastungsfaktoren auf der Klassen- und Schulebene zu untersuchen. Insbesondere sollte dabei die Frage beantwortet werden, ob die Arbeitsunzufriedenheit und -überforderung von Lehrkräften auch mit verhaltensbezogenen Merkmalen der unterrichteten Schulkinder in Verbindung gebracht werden können. Die Analysen wurden mit national-repräsentativen Daten von Lehrpersonen der 5. bis 9. Klasse aus allen Landesteilen der Schweiz durchgeführt.

Als Hauptergebnis der Untersuchung kann festgehalten werden, dass Variablen, die sich auf problematisches Verhalten der unterrichteten Schulkinder beziehen, weder mit der subjektiv wahrgenommenen Arbeitsunzufriedenheit noch mit der Arbeitsüberforderung der unterrichtenden Lehrperson in Zusammenhang stehen. Dieser Befund steht in scheinbarem Widerspruch zu Ergebnissen eingangs zitierter kantonaler Studien, die aufzeigen, dass von den Lehrpersonen das Problemverhalten von unterrichteten Jugendlichen als starke Belastung empfunden wird (Nido et al., 2008; Trachsler et al., 2003; Trachsler et al., 2006). Allerdings muss beachtet werden, dass bei diesen Studien die Lehrpersonen direkt nach konkreten, in ihrer Arbeitssituation möglichen Belastungsaspekten befragt wurden. Für die Analysen in der vorliegenden Arbeit wurden demgegenüber Aggregatdaten aus den Angaben der unterrichteten Schulkinder verwendet. Wie bereits erläutert lässt sich aufgrund dieser Methodik eine rekursive Beeinflussung der verwendeten Indikatoren durch den Grad der Arbeitsunzufriedenheit oder -überforderung der Lehrperson praktisch ausschliessen.

Relevante Zusammenhänge mit der Arbeitsunzufriedenheit und -überforderung sind aber bei strukturellen Merkmalen der Klasse und schulbezogenen Variablen zu finden. Bei der Arbeitsunzufriedenheit deuten die Ergebnisse darauf hin, dass insbesondere die erfahrene Unterstützung durch die Schule bzw. durch die Eltern von Bedeutung ist - sofern man davon ausgeht, dass die spezifische Unterstützung in den Bereichen Gesundheitserziehung und -förderung auch mit der generell erfahrenen Unterstützung korrelieren dürfte. Bei der Arbeitsüberforderung scheint die Klassengrösse einen - allerdings eher geringen - Einfluss zu ha- 
ben.

Interessanterweise zeigte sich ein negativer Zusammenhang zwischen der Höhe der unterrichteten Schulstufe und der subjektiv wahrgenommenen Arbeitsunzufriedenheit bzw. -überforderung. Über die Gründe dafür kann hier nur spekuliert werden. So ist etwa denkbar, dass Lehrpersonen höherer Stufen von grösserer sozialer Anerkennung, einer besseren Entlöhnung und tendenziell kleineren Arbeitspensen profitieren können, und sich deshalb weniger überfordert und/oder unzufrieden fühlen. Diese Annahmen konnten aber mit den hier verwendeten Daten nicht verifiziert werden, ebenso wenig wie mögliche Differenzen zwischen Primar-, Real-, und Sekundarschullehrpersonen. Es sei hier auch noch einmal daran erinnert, dass die der Stichprobe zugrunde liegende Grundgesamtheit nur Lehrkräfte der 5. bis 9. Schulstufen umfasst.

Bei den demografischen Variablen steht der positive Zusammenhang zwischen Alter und subjektiv wahrgenommener Arbeitsunzufriedenheit bzw. -überforderung in Einklang mit ähnlichen Befunden aus früheren Schweizer Studien (Bieri, 2002; Landert, 2007). Zur Erklärung dieses Zusammenhangs kann in verschiedene Richtungen argumentiert werden. Zum Einen dürfte es für ältere Lehrpersonen schwieriger sein, sich ändernden Erwartungen und Lehrplänen anzupassen. Zum Anderen liegt auch die Vermutung eines Selektionsprozesses nahe: Während jüngere Lehrerinnen und Lehrer, die unter Arbeitsunzufriedenheit oder überforderung leiden, relativ einfach aus dem Lehrberuf aussteigen und in einem anderen Arbeitsumfeld einen Quereinstieg wagen können, ist ein Berufswechsel für ältere Lehrpersonen schwieriger. In dieser auf Querschnittsdaten basierten Studie lassen sich solche Vermutungen jedoch nicht belegen.

Die Lehrpersonenbefragung im Rahmen der HBSC-Studie liefert aktuell die einzigen national-repräsentativen Daten, die auch direkte Vergleiche zwischen den Sprachregionen zulassen. Diese müssen gemäss den Ergebnissen dieser Arbeit als gewichtiger Einflussfaktor für die Arbeitsüberforderung angesehen werden: Lehrerinnen und Lehrer geben in der lateinischen Schweiz eine bedeutend höhere Arbeitsüberforderung an als in der Deutschschweiz. Zu diesem Ergebnis kam bereits die Analyse der Lehrpersonenbefragung im Rahmen der HBSC-Studie 2002 (Delgrande Jordan et al., 2005). Bei der Arbeitsunzufriedenheit spielt die Sprachregion hingegen nur insofern eine Rolle, als dass sich in der lateinischen Schweiz ein Zusammenhang mit der erfahrenen Unterstützung der Schule zeigt, der in der Deutschschweiz nicht vorhanden ist. Die sprachregionalen Einflüsse dürften mit strukturellen Unterschieden in den Bereichen der Ausbildung der Lehrpersonen und der Schul- und Unterrichtsorganisation zusammenhängen, die in unserer Untersuchung nicht erfasst werden konnten.

Dass schulbezogene Faktoren in bedeutendem Masse mit dem beruflichen Befinden von Lehrpersonen in Zusammenhang stehen können, zeigt sich in unserer Untersuchung vor allem in Bezug auf die subjektiv wahrgenommene Arbeitsunzufriedenheit und die erfahrene Unterstützung von Seiten der Eltern und der Schule. Die Ergebnisse mit Einbezug von Interaktionstermen zeigen sowohl 
für Arbeitsunzufriedenheit als auch für die Arbeitsüberforderung zudem, dass die erfahrene Unterstützung gerade für diejenigen Gruppen von Lehrpersonen von erhöhter Bedeutung ist, welche ein grösseres Risiko aufweisen, von Arbeitsunzufriedenheit oder -überforderung betroffen zu werden. Es sind namentlich die eher älteren Lehrpersonen sowie Lehrerinnen und Lehrer in der lateinischen Schweiz, die besonders auf solche Unterstützungen angewiesen zu sein scheinen.

\section{Einschränkungen und Ausblick}

In der vorliegenden Arbeit wurden erstmalig für die Schweiz Zusammenhänge zwischen subjektiv wahrgenommener Arbeitsunzufriedenheit bzw. -überforderung von Lehrpersonen und möglichen Einflussfaktoren anhand einer nationalrepräsentativen Zufallsstichprobe untersucht. Mit einer Reihe demografischer, klassen- und schulbezogener Variablen gingen dabei potentielle Einflussfaktoren verschiedener Ebenen in die Analysen ein. Als methodische Innovation wurden für verhaltensbezogene (klassenbezogene) Variablen auch Ergebnisse aus einer Befragung der unterrichteten Schulklasse miteinbezogen, die somit tatsächlich als von der Arbeitsunzufriedenheit bzw. überforderung der Lehrperson unabhängig gelten können. Neben diesen wesentlichsten Stärken soll im Folgenden auch auf einige Schwächen dieser Arbeit hingewiesen sein, sowie ein Ausblick auf mögliche Ansätze in weiterführenden Studien und präventive Massnahmen für die Gesundheitserhaltung von Lehrpersonen gewährt werden.

Zunächst sei daran erinnert, dass die in dieser Arbeit aufgezeigten $\mathrm{Zu}-$ sammenhänge allgemein nicht als kausale Effekte interpretiert werden können, da ihnen lediglich Querschnittsdaten zugrunde liegen.

Ausserdem gilt es zu beachten, dass die verwendeten schulbezogenen Variablen aus der Befragung der Lehrpersonen entnommen wurden. Es ist also möglich, dass diese Angaben von der subjektiv wahrgenommenen Arbeitsunzufriedenheit und -überforderung der Lehrperson nicht unabhängig sind. So kann argumentiert werden, dass bereits unzufriedene Lehrerinnen und Lehrer ein bestimmtes Niveau an gebotener Unterstützung der Schule bzw. Eltern als geringer einschätzen oder wahrnehmen als Lehrpersonen mit tieferer Arbeitsunzufriedenheit. Die diesbezüglich aufgezeigten Zusammenhänge könnten durch einen solchen Effekt überschätzt worden sein. In zukünftigen Studien sollte deshalb versucht werden, diese - und eventuell weitere strukturelle Merkmale der Schule von den Lehrpersonen unabhängig zu erfassen, um nach dem gleichen Prinzip wie bei den verhaltensbezogenen Variablen der Schulklasse einen solchen Effekt ausschliessen zu können.

Bei diesen ihrerseits auf der direkten Befragung der unterrichteten Klasse basierten verhaltensbezogenen Variablen liesse sich vermuten, dass allfällige $\mathrm{Zu}$ sammenhänge mit der Arbeitsunzufriedenheit und/oder -überforderung aufgrund teilweise geringer Interaktionsopportunitäten zwischen Schulklasse und 
Lehrperson in dieser Arbeit unterschätzt worden seien. Ergänzende Analysen haben diesbezüglich aber gezeigt, dass bei einer Beschränkung auf eine Teilstichprobe von 276 Lehrpersonen, die die befragte Klasse mindestens 16 Stunden pro Woche unterrichten, ebenfalls keine signifikanten diesbezüglichen Zusammenhänge beobachtet werden können.

Ausserdem stellt sich die Frage, wie stark allgemeine Problemverhalten (Jessor $\&$ Jessor, 1977) unter den Schülerinnen und Schülern auch auf problematische Verhaltensweisen im Schulzimmer hindeuten. Obwohl hier ein starker $\mathrm{Zu}-$ sammenhang vermutet werden darf, wäre es doch wünschenswert, auch Indikatoren für das Ausmass problematischen Verhaltens während des Unterrichts in zukünftige Studien einzubeziehen.

Insgesamt können die in dieser Analyse aufgezeigten Zusammenhänge nur einen kleinen Anteil der Varianz der abhängigen Variablen (korrigiertes $\mathrm{R}^{2}$ im Modell mit Interaktionsthermen für Arbeitsunzufriedenheit $=7.2 \%$, für Arbeitsüberforderung $=8.8 \%$ ) erklären. Subjektiv wahrgenommene Arbeitsunzufriedenheit und -überforderung von Lehrpersonen scheinen sehr komplexe Phänomene zu sein, deren Einflussfaktoren schwierig zu bestimmen sind. Aus der Sicht dieser explorativen Arbeit kann jedoch gesagt werden, dass in weiterführenden Studien vor allem versucht werden sollte, weitere strukturelle Faktoren einzubeziehen, die in direktem Zusammenhang mit dem Arbeitsumfeld und der Arbeitsweise der individuellen Lehrperson stehen. Insbesondere der Einbezug von Variablen für das Unterrichtspensum, das Salär, die Stellung der Lehrperson im Kollegium sowie die Durchführung von Reformen und die Belastung durch administrative Arbeiten scheinen hier für eine weitergehende Erklärung der Arbeitsunzufriedenheit und -überforderung vielversprechend (Albisser et al., 2006; Bieri, 2002; Landert, 2007).

Das psychische Wohlbefinden der Lehrpersonen ist nicht nur für deren eigene Gesundheit von grosser Wichtigkeit, sondern auch für das Wohlergehen der unterrichteten Schulkinder (Klusmann et al., 2006; Klusmann et al., 2008). Die Ergebnisse dieser Studie deuten darauf hin, dass zu deren Erhaltung und Förderung am ehesten Massnahmen im Bereich der Arbeitsweise und des Arbeitsumfelds an der Schule angebracht wären. Gerade in der lateinischen Schweiz und für ältere Lehrpersonen scheint die wahrgenommene Unterstützung von Seiten der Schule bzw. der Eltern eine Rolle zu spielen. Diese kann durch institutionalisierte Unterstützungssysteme für Lehrpersonen sowohl innerhalb des Lehrkollegiums als auch in einem Netzwerk zwischen Lehrpersonen, den Schulbehörden und den Eltern verbessert werden und somit zu einer guten und gesunden Schule beitragen (Freitag, 1998; Paulus, 2003). 


\section{Anmerkungen}

1 Die Lehrpersonenstudie wurde im Rahmen der Studie „Health Behaviour in School-aged Children (HBSC)“ durchgeführt (Bundesamt für Gesundheit, Vertrag Nr. 04.001776). Die Auswertung der Lehrpersonenstudie wurde durch Eigenmittel von Such Info Schweiz finanziert.

2 Der genaue Wortlaut der Items lautet:

Arbeitsunzufriedenheit: 1.) Mein Beruf macht mir Spass; 2.) Ich habe mir schon ernsthaft überlegt, aus dem Beruf auszusteigen; 3.) Was meine Arbeit betrifft, bin ich eigentlich rundum zufrieden; 4.) Ich halte mich gern an meinem Arbeitsplatz auf; 5.) Ich kann in meinem Beruf meine Fähigkeiten voll nutzen; 6.) Meine eigenen Berufsideale lassen sich in meiner Tätigkeit verwirklichen.

Arbeitsüberforderung: 1.) In meinem Beruf wird man ständig überfordert; 2.) Ich fühle mich häufig überfordert; 3.) Ich habe selten das Gefühl, einmal richtig abschalten zu können; 4.) Oft habe ich ein schlechtes Gewissen den Schülerinnen und Schülern gegenüber; 5.) Für andere Menschen verantwortlich zu sein, belastet mich sehr; 6.) Der Zeitdruck, unter dem ich arbeite, ist zu gross.

3 Bei den umgekehrt formulierten Items 1, 3, 4, 5 und 6 für die Arbeitsunzufriedenheit (siehe Anmerkung 2) lauten die entsprechenden Antworten „trifft völlig zu“ (=1), „trifft überwiegend zu“ (=2) oder „trifft mittelmässig zu“ (=3).

\section{Literatur}

Albisser, S., Kirchhoff, E., Meier, A. \& Grob, A. (2006, Dezember). Anforderungsverarbeitung und Gesundheit im Berufszyklus von Lehrpersonen. Paper vorgestellt an der Fachtagung "Balancieren im Lehrberuf". Bern.

Bachmann, K. (1999). Lust oder Last - Berufszufriedenheit und Belastung im Beruf bei Lehrerinnen und Lehrern an berufsbildenden Schulen. Baltmannsweiler: Schneider Verlag Hohengehren $\mathrm{GmbH}$.

Backhaus, K., Erichson, B., Plinke, W. \& Weiber, R. (2000). Multivariate Analysemethoden Eine anwendungsorientierte Einführung (9. Aufl.). Berlin: Springer-Verlag.

Bieri, T. (2002). Die berufliche Situation aus der Sicht der Lehrpersonen - Zufriedenheit, Belastung, Wohlbefinden und Kündigungen im Lehrberuf. Dissertation, Eberhard-Karls-Universität, Tübingen.

Bucher, B. (2001). Arbeitszeit und Arbeitsbelastung der Lehrpersonen - Einsichten, Aussichten, Folgerungen (Literaturbericht). Luzern: Bildungsplanung Zentralschweiz.

Delgrande Jordan, M., Kuntsche, E. \& Sidler, J. (2005). Arbeitsüberforderung und -unzufriedenheit von Lehrpersonen in der Schweiz - Zusammenhänge mit Depressivität und somatischen Beschwerden. Schweizerische Zeitschrift für Bildungswissenschaften, 27 (1), 123-139.

Enzmann, D. \& Kleiber, D. (1989). Helfer-Leiden: Stress und Burnout in psychosozialen Berufen. Heidelberg: Asanger.

Freitag, M. (1998). Was ist eine gesunde Schule? Einflüsse des Schulklimas auf Schüler-und Lehrergesundheit. Weinheim: Juventa Verlag.

Gilks, W. R., Richardson, S. \& Spiegelhalter, D. J. (Ed.). (1996). Markov Chain Monte Carlo in practice. Interdisciplinary statistics. London: Chapman \& Hall.

Gonik, V., Kurth, S. \& Boillat, M.-A. (2000). Analyse du questionnaire sur l'état de santé physique et mentale des enseignants vaudois (Rapport final). Lausanne: Institut universitaire romand de Santé au Travail (IST).

Ipfling, H. J., Peez, H. \& Gamsjäger, E. (1995). Wie zufrieden sind die Lehrer? Bad Heilbrunn: Klinkhardt.

Jaccard, J., Turrisi, R. \& Wan, C. K. (1990). Interaction effects in multiple regression. Newbury 
Park, CA: Sage.

Jarvis, M. (2002). Teacher stress: A critical review of recent findings and suggestions for future research directions. Stress News, 4 (1), 1-7.

Jessor, R. \& Jessor, S. L. (1977). Problem behavior and psychosocial development: A longitudinal study of youth. New York: Academic Press.

Jöreskog, K. G. \& Sörbom, D. (2001). LISREL Software Version 8.51. Chicago, IL: Scientific Software International, Inc.

Klusmann, U., Kunter, M., Trautwein, U. \& Baumert, J. (2006). Lehrerbelastung und Unterrichtsqualität aus der Perspektive von Lehrenden und Lernenden. Zeitschrift für Pädagogische Psychologie, 20 (3), 161-173.

Klusmann, U., Kunter, M., Trautwein, U., Lüdtke, O. \& Baumert, J. (2008). Engagement and emotional exhaustion in teachers: Does the school context make a difference? Applied Psychology: An International Review, 57, 127-151.

Kuntsche, E. (2004). Progression of a general substance use pattern among adolescents in Switzerland? Investigating the relationship between alcohol, tobacco, and cannabis use over a 12-year period. European Addiction Research, 10 (3), 118-125.

Kuntsche, E., Delgrande Jordan, M. \& Sidler, J. (2005). Rauchen und trinken Lehrpersonen täglich (mehr), wenn sie arbeitsüberfordert und -unzufrieden sind? Abhängigkeiten, 11 (1), 52-65.

Landert, C. (2007). Die Berufszufriedenheit der Deutschschweizer Lehrerinnen und Lehrer: Bericht über die Onlinebefragung vom September/Oktober 2006. Zürich: Landert Farago \& Partner.

Merz, J. (1979). Berufszufriedenheit von Lehrern. Eine empirische Untersuchung. Weinheim: Beltz.

Nido, M., Ackermann, K., Ulich, E., Trachsler, E. \& Brüggen, S. (2008). Arbeitsbedingungen, Belastungen und Ressourcen von Lehrpersonen und Schulleitungen im Kanton Aargau 2008 Ergebnisse der Untersuchung im Auftrag des Departements Bildung, Kultur und Sport (BKS, Kanton Aargau). Zürich: Institut für Arbeitsforschung und Organisationsberatung (iafob).

Paulus, P. (2003). Schulische Gesundheitsförderung - vom Kopf auf die Füsse gestellt. Von der Gesundheitsfördernden Schule zur guten, gesunden Schule. In K. Aregger \& U. P. Lattmann (Hrsg.), Gesundheitsfördernde Schule - eine Utopie? Konzepte, Praxisbeispiele, Perspektiven (S. 93-114). Aarau: Sauerländer Verlag.

Rudow, B. (1994). Die Arbeit des Lehrers. Zur Psychologie der Lehrertätigkeit, Lehrerbelastung und Lehrergesundheit. Bern: Hans Huber.

Schaarschmidt, U. (Hrsg.). (2005). Halbtagsjobber? Psychische Gesundheit im Lehrerberuf Analyse eines veränderungsbedürftigen Zustandes. Weinheim: Beltz.

Schafer, J. L. (1997). Analysis of incomplete multivariate data. London: Chapman \& Hall.

Schmid, H., Kuntsche, E. \& Delgrande, M. (Hrsg.). (2001). Anpassen, ausweichen, auflehnen? Fakten und Hintergründe zur psychosozialen Gesundheit und zum Konsum psychoaktiver Substanzen von Schülerinnen und Schülern. Bern: Haupt.

Schmitz, G. S. (1999). Zur Struktur und Dynamik der Selbstwirksamkeitserwartung von Lehrern. Ein protektiver Faktor gegen Belastung und Burnout? Unveröffentlichte Dissertation, Freie Universität Berlin.

Schwarzer, R. \& Jerusalem, M. (Hrsg.). (1999). Skalen zur Erfassung von Lehrer- und Schülermerkmalen. Dokumentation der psychometrischen Verfahren im Rahmen der Wissenschaftlichen Begleitung des Modellversuchs Selbstwirksame Schulen. Berlin: Freie Universität Berlin.

Spear, M., Gould, K. \& Lee, B. (2000). Who would be a teacher? A review of factors motivating and demotivating prospective and practising teachers. Slough: NFER.

Trachsler, E., Brüggen, S., Nido, M., Ulich, E., Inversini, S., Wülser, M. \& Herms, I. (2006). Arbeitsbedingungen, Belastungen und Ressourcen in der Thurgauer Volksschule - Teilstudie Lehrkräfte Ergebnisse der zweiten Erhebung 2005 (Forschungsbericht Nr. 4). Kreuzlingen / Zürich: Pädagogische Hochschule Thurgau / Institut für Arbeitsforschung und Organisationsberatung. 
Trachsler, E., Ulich, E., Inversini, S. \& Wülser, M. (2003). Arbeitsbedingungen, Belastungen und Ressourcen der Thurgauer Volksschullehrkräfte angesichts der laufenden Bildungsoffensive - Ergebnisse der Analyse der ersten Teilstudie. Kreuzlingen / Zürich: Pädagogische Hochschule Thurgau / Institut für Arbeitsforschung und Organisationsberatung.

Ulich, E., Inversini, S. \& Wülser, M. (2002). Arbeitsbedingungen, Belastungen und Ressourcen der Lehrkräfte des Kantons Basel-Stadt. Zürich: Institut für Arbeitsforschung und Organisationsberatung.

Schlüsselwörter: Arbeitszufriedenheit, Berufsbelastung, Lehrpersonen

\section{Surcharge et insatisfaction professionnelles chez les enseignants - Résultats représentatifs pour la Suisse de facteurs démographiques et relatifs à la classe et à l'école}

\section{Résumé}

Sur la base d'un échantillon représentatif pour la Suisse, les liens entre, d'une part, la surcharge et l'insatisfaction professionnelles perçues par les enseignants et, d'autre part, des facteurs démographiques et relatifs à la classe et à l'école sont étudiés. Les réponses de 534 enseignants de $5^{\mathrm{e}}$ à $9^{\mathrm{e}}$ années et de leurs élèves sont analysées au moyen de régressions linéaires multiples. Aucun lien n'est observé entre les comportements problématiques des élèves et la surcharge et l'insatisfaction professionnelles perçues par les enseignants. En revanche, en plus des caractéristiques démographiques des enseignants, le soutien qui leur est apporté par l'école et par les parents, de même que d'autres caractéristiques structurelles de l'école et de la classe, semblent jouer un rôle dans l'explication de la surcharge et de l'insatisfaction professionnelles.

Mots clés: Satisfaction professionnelle, charge professionnelle, enseignants

\section{Sovraccarico e insoddisfazione professionale dei docenti in Svizzera - risultati rappresentativi a livello nazionale per fattori demografici e relativi alla classe e alla scuola}

\section{Riassunto}

Questo studio esplora il legame tra il sovraccarico e l'insoddisfazione professionale percepite dagli insegnanti, e fattori demografici e relativi alla classe e alla scuola in un campione rappresentativo per la Svizzera. Le riposte dei 534 insegnanti del $5^{\circ}$ e $9^{\circ}$ anno e dei loro allievi sono state analizzate tramite regressioni lineari multiple. Non è stato rilevato alcun legame tra i comportamenti proble- 
matici degli allievi e il sovraccarico e l'insoddisfazione professionali percepita dai docenti. Al contrario, oltre alle caratteristiche demografiche degli insegnanti, le caratteristiche strutturali della scuola e della classe sembrano giocare un ruolo nello spiegare il sovraccarico e l'insoddisfazione professionali.

Parole chiave: Soddisfazione professionale, carico professionale, docenti

\section{Professional overload and job dissatisfaction among teachers in Switzerland - representative results for demographic and class and school related factors}

\section{Abstract}

Based on a representative sample of teachers in Switzerland, this paper investigates associations between perceived professional overload and job dissatisfaction, and demographic and class as well as school related characteristics. Using multiple linear regressions, the answers of 534 fifth-to-ninth grade teachers and their classes were analysed. For problematic behaviour of the pupils, no association was found neither with perceived professional overload nor with job dissatisfaction of the teacher. In contrast, besides demographic variables, the support offered by the school and the parents seem to account for perceived professional overload and job dissatisfaction of teachers.

Key words: Professional satisfaction, workload, teachers 